\title{
Entropía de grafos y su uso para medir la inteligibilidad de la ciudad ${ }^{1}$ \\ Graph entropy as a means of measuring the intelligibility of a city
}

\author{
Antonio Aguilera Ontiveros* y Edgardo Ugalde Saldaña**
}

\section{INTRODUCCIÓN}

Los seres humanos nos movemos dentro de nuestros ambientes urbanos basados principalmente en la construcción de mapas cognitivos (Haq y Girotto, 2003). Se han identificado tres clases de relaciones espaciales que forman el contenido de la cognición espacial, éstas relaciones son de tipo topológico, proyectivo y métrico o euclidiano (Haq y Girotto, 2003). Además, se ha encontrado que el entendimiento de las relaciones topológicas precede el entendimiento de las relaciones proyectivas y euclidianas (Haq y Girotto, 2003). Shemyakin (1962), citado por Haq y Girotto, (2003), menciona que existen dos tipos de representaciones topológicas: los mapas de ruta y los mapas de reconocimiento. Los primeros son construidos a través del trazo mental de la ruta de movimiento a través de un área, mientras que los segundos son representaciones de la configuración general de la mutua disposición de los objetos locales. De lo anterior se deriva una distinción entre las variables topológicas locales y las ideas topológicamente derivadas acerca de la configuración general del entorno.

\footnotetext{
${ }^{1}$ Este trabajo fue parcialmente apoyado por el Consejo Nacional de Ciencia y Tecnología de México a través del proyecto 101366. Nuestro agradecimiento.

*El Colegio de San Luis, A. C. (aaguilera@colsan.edu.mx).

** Instituto de Física, Universidad Autónoma de San Luis Potosí (ugalde@ifisica.uaslp.mx).
} 
Lynch (1997) contribuye al estudio de la cognición del espacio a través de su idea de legibilidad, la cual se entiende como la facilidad con la que las partes de una ciudad pueden ser reconocidas y se pueden organizar en un patrón coherente. Por su parte Hillier (1996) contribuyó a la discusión con su concepto de la inteligibilidad, la cual se relaciona con la orientación y la búsqueda de rutas (wayfinding) en un espacio. Hillier (1996) establece que la inteligibilidad significa el grado de lo que podemos ver de los espacios que conforman el sistema (means the degree to which what we can see from the spaces that make up the system). La idea anterior fue desarrollada por Hillier en lo que se llegó a convertir en la teoría de la sintaxis espacial.

Este trabajo tiene como finalidad discutir la definición de Hillier (1996) sobre la inteligibilidad del espacio y mostrar un método para calcular la inteligibilidad, basado en la teoría de grafos, el cual fue propuesto por Volchenkov y Blanchard (2008). Para ilustrar el método se muestra el estudio que se le realizó a la red de calles que conforman el centro histórico y sus alrededores, de la zona metropolitana de San Luis Potosí, México. La estructura de este trabajo está desarrollada de la siguiente manera: en la sección siguiente, se discute los aspectos teóricos referentes a la sintaxis del espacio y las propuestas de medición de la inteligibilidad sugeridas por Hillier (1996) y Volchenkov y Blanchard (2008). Después se exponen los fundamentos matemáticos de la teoría de grafos y la entropía de grafo y la forma en que ésta es usada por Volchenkov y Blanchard para calcular la inteligibilidad. En seguida se desarrolla el método de estudio de la red formada por las calles del centro de la ciudad de San Luis Potosí. La última sección está dedicada a mostrar los resultados obtenidos en el caso y las reflexiones finales con respecto al método trabajado.

\section{LA SINTAXIS DEL ESPACIO Y LA INTELIGIBILIDAD}

La sintaxis del espacio es un conjunto de teorías y técnicas para el análisis de configuraciones espaciales. Se basa en la visión de que la estructura espacial es un conjunto de interconexiones entre espacios definidos y diferenciados, pero que al mismo tiempo son interpretados por los seres humanos en su interacción con los mismos (Hillier, 1996). La relevancia de la sintaxis del espacio para el estudio de la ciudad reside en que permite visualizar y comprender no solo los aspectos morfológicos de las urbes, sino también el estudio de los procesos de cognición del espacio desarrollados por los seres humanos y los comportamientos que emergen de dichos procesos (Hillier y Hanson, 1984). 
Uno de los conceptos teóricos de la sintaxis del espacio es la inteligibilidad. Ésta es la propiedad que tiene el espacio urbano de ser comprendido por las personas que lo transitan y/o habitan. La inteligibilidad les permite a las personas acceder a información no local sobre la configuración del espacio, permitiéndoles desarrollar mapas cognitivos que les permitan encontrar rutas para sus desplazamientos. Hillier (1996) definió la inteligibilidad como la correlación entre la integración del espacio y la conectividad del mismo. Lo que en términos generales implica una correlación entre propiedades globales y locales del espacio.

Para poder llegar a definir formalmente los conceptos clave definidos por Hillier para medir la inteligibilidad es necesario introducir una serie de conceptos desarrollados dentro de la teoría de la sintaxis del espacio. A esto nos dedicaremos en los siguientes párrafos.

La sintaxis del espacio está lejanamente emparentada con la teoría de grafos y la combinatoria, esto es importante señalarlo, ya que la sintaxis del espacio desarrolla sus propios conceptos los cuales podrían ser interpretados de una forma más sólida desde la teoría de grafos.

La herramienta principal de la sintaxis del espacio es el mapa axial. Éste es un conjunto mínimo de líneas rectas de la mayor longitud posible que cruza e interconecta los espacios abiertos de un sistema urbano (Hillier y Hanson, 1984). La descomposición del mapa axial crea un grafo en el cual los nodos son líneas y las intersecciones son arcos. A partir de dicho grafo se establecen medidas de tipo topológico para cuantificar las características del espacio urbano.

La integración de un espacio urbano se entiende en la sintaxis del espacio como la cuantificación de la profundidad del mapa axial. La profundidad es la distancia de una línea hacia las otras. En términos prácticos, lo que se mide es cuantos "pasos» sobre el grafo hay que dar para moverse entre diferentes lugares del espacio urbano. Un valor alto de integración establece que los espacios axiales están bien conectados y el movimiento es fácil entre ellos, por el contrario un bajo valor de integración nos estaría hablando de aislamiento espacial.

Veamos ahora un ejemplo sencillo de lo que hemos explicado anteriormente. En la figura 1 se muestra un espacio conformado por ocho cuartos interconectados entre si. En la parte izquierda se muestra el grafo correspondiente a dicho espacio, mostrando la profundidad que tiene cada cuarto (espacio) con respecto a la lógica de su transito. Nótese que el nodo 1 es el nodo de inicio del recorrido. Para ir a cualquier nodo es necesario pasar por uno. También nótese que los nodos del 2 al 6 son accesibles desde el nodo 1, sin em- 
bargo, los nodos 7 y 8 necesitan un nodo intermedio para poder acceder a los mismos, esto es, se necesita pasar por 3 para alcanzar 7 y por 4 para alcanzar 8 . La profundidad es el número de pasos (nodos) que tenemos que usar para llegar a cualquiera. En este caso la profundidad se midió desde el nodo 1. Si por ejemplo comenzáramos desde el nodo 5 para ir al tres el camino sería 5-1-3, lo que daría que 3 quedara con una profundidad 2 para este caso. Nótese entonces que la profundidad no es una cantidad absoluta sino relativa.

\section{FIGURA 1}

MAPA AXIAL DE UN ESPACIO Y LA PROFUNDIDAD DE SUS COMPONENTES

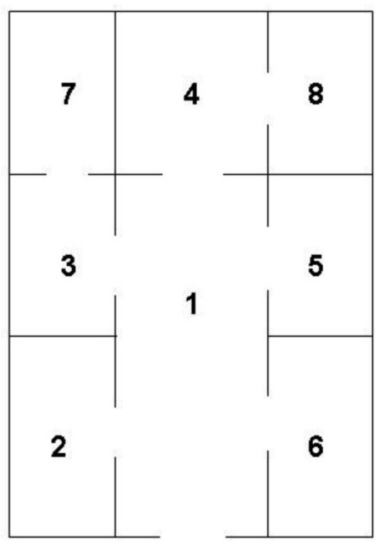

Profundidad

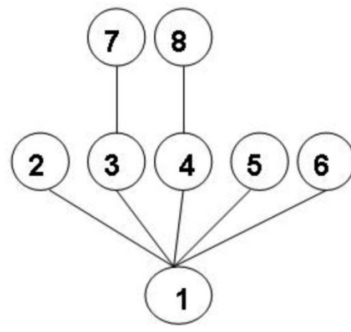

2

1

0

Fuente: elaboración propia.

La simetría de un espacio construido refleja el grado de integración con el resto del espacio. Se cuantifica midiendo la profundidad de un espacio desde los demás espacios que conforman el sistema espacial. El valor de asimetría relativa compara la profundidad media de la estructura a partir de un espacio dado con la profundidad máxima que podría una estructura con el mismo número de componentes. Valores bajos de la asimetría relativa, esto es entre cercanos a cero, representan un espacio integrador.

La asimetría relativa se calcula a través de la siguiente formula (Hillier y Hanson, 1984):

$$
A_{R}=\frac{2(M D-1)}{k-2}
$$


En donde $M D$ es la profundidad media de la estructura espacial y $k$ el número de espacios en que la componen.

La profundidad media para un sistema espacial parte del establecimiento de un punto dado del mismo, asignando valores a los demás espacios del sistema dependiendo de los pasos que los separe del punto original previamente establecido. Todos los espacios adyacentes al punto tendrán profundidad 1 , los siguientes tendrán profundidad 2, etcétera. La profundidad promedio del punto elegido se calcula sumando los valores promedio y dividiendo por el número de espacios $k$ del sistema menos uno, lo que significa remover el espacio original.

Los valores de la asimetría relativa se encuentran entre 0 y 1 , siendo 0 el valor al que le corresponde la máxima integración del espacio.

La integración es la primera variable sugerida por Hillier para medir la inteligibilidad. La segunda variable es la conectividad, la cual es definida como el número de espacios que están conectados directamente con un espacio dado. La conectividad es una medida de tipo local. La correlación positiva entre estas dos variables nos da la medida de inteligibilidad del espacio.

Volchenkov y Blanchard (2008) han propuesto un método basado en la entropía de grafos para medir la inteligibilidad del espacio urbano. Dicho método difiere de la forma anteriormente expresada y establecida en el trabajo de Hillier, pero preserva la esencia de que la inteligibilidad de un espacio codificado por una red es la correlación entre dos medidas topológicas una local y una global. El método no necesita la construcción de un mapa axial, éste es sustituido por un grafo que representa la interconexión entre los espacios abiertos (calles) de un entorno urbano. Dicho grafo se construye considerando no las intersecciones de las calles como los nodos de la red, sino tomando como nodos los nombres de las calles y las interconexiones se dan cuando dos calles se intersectan.

Volchenkov y Blanchard (2008) y Blanchard y Volchenkov (2010) proponen el usar la entropía de grafos para calcular a cada vértice su tasa de participación en la entropía total del grafo. La entropía de grafos es medida a través de la definición de dos distribuciones de probabilidad. La primera fundamentada en la conectividad de los vértices y la segunda basada en la centralidad de los vértices. La primera distribución es una medida local y la segunda es una medida global. El cálculo de la correlación de Pearson permite medir la inteligibilidad del espacio urbano en cuestión.

En la siguiente sección se desarrollan los aspectos matemáticos para entender el método de Volchenkov y Blanchard. 
TEORÍA DE GRAFOS Y ENTROPÍAS DE GRAFOS

En este aparatado definiremos los aspectos básicos de la teoría de grafos que usaremos en el método de cálculo de la inteligibilidad propuesto por Volchenkov y Blanchard.

Una red puede ser representada matemáticamente como una dupla $G(V, E)$ a través del uso de dos conjuntos. El primero es un conjunto no vacío y finito de vértices o nodos $V$ y el segundo es un conjunto no vacío y finito de $\operatorname{arcos} E$ que relacionan un elemento de $V$ con otro elemento de $V$, no necesariamente distinto. Formalmente, cada $e \in E$ está asociado a una dupla $\left(v_{i}, v_{j}\right) \in V \times V$. En nuestro estudio, nosotros estamos interesados solamente en grafos no dirigidos sin lazos, esto es, si $e \in E$ está asociado con $\left(v_{i}, v_{j}\right)$, entonces también está asociado con $\left(v_{i}, v_{j}\right)$ y además siempre $i \neq j$.

Un recorrido sobre un grafo es una sucesión de vértices que están interconectados por pares (asociados a un arco) de tal forma que existe una colección de vértices que permiten ir de un vértice $v_{i}$ a un vértice $v_{k}$. La longitud del recorrido es el número de vértices que hay que pasar para ir del vértice $i$ al vértice $k$. Nótese que puede haber más de un recorrido que conecte dos vértices cualesquiera en un grafo. El camino más corto entre dos vértices $v_{i}$ y $v_{j}$ es aquel en dónde la longitud es mínima en función de las longitudes de todos los recorridos que conectan a los vértices $v_{i}$ y $v_{j}$. Al camino más corto también se le conoce como geodésica.

Dado un grafo con un conjunto de vértices $\mathrm{V}$, el grado de un vértice es el número de arcos asociados a duplas en $V \times V$ que contiene a $v$ y es simbolizado por $\operatorname{deg}(v)$.

La intermediación (betweenness) es otra medida de grafos relevante. Esta fue definida por Freeman (1977) como sigue:

Dado un vértice $\in V$ en un grafo $G(V, E)$ y dada una dupla vértices $\left(v_{i}, v_{j}\right)$, donde $i \neq j$, la intermediación parcial $b_{i j}\left(v_{k}\right)$ es el número de caminos más cortos que conectan $v_{i}$ con $v_{j}$ y que pasan a través del vértice $v_{k}$, dividido por el total de caminos más cortos que conectan $v_{i} \operatorname{con} v_{j}$. Por lo tanto, sí $g_{i j}\left(v_{k}\right)$ denota el número de caminos más cortos que conectan $v_{i} \operatorname{con} v_{j}$ y que pasan por $v_{k}$, y $g_{i j}$ denota el número total de caminos cortos que conectan $v_{i} \operatorname{con} v_{j}$, entonces la intermediación sería:

$$
b_{i j}\left(v_{k}\right)=g_{i j}\left(v_{k}\right) / g_{i j}
$$

Dicha ecuación se entiende como la probabilidad de que un vértice $v_{k}$ pertenezca a una geodésica aleatoriamente seleccionada que conecta $v_{i} \operatorname{con} v_{j}$. 
La centralidad total de un vértice $v_{k}$ es definida como la suma de sus intermediaciones parciales para todos los pares no ordenados de vértices:

$$
C_{B}\left(v_{k}\right)=\sum_{i<j}^{N} \sum_{j}^{N} b_{i j}\left(v_{k}\right)
$$

Donde $\mathrm{i} \neq \mathrm{j} \neq \mathrm{k}$, y $\mathrm{N}$ es el número total de vértices en el grafo.

Una vez entendidos los conceptos anteriores de la teoría de grafos, pasaremos a entender el concepto de entropía de grafo. Para esto es necesario primero explicar el concepto de entropía. La entropía es un parámetro usado para describir la incertidumbre de un sistema. Dicho parámetro se construye a partir de la medición de la distribución de probabilidad de los estados en que puede estar un sistema. En 1948, Shannon propuso la siguiente formulación general de la entropía:

$$
S(\Omega, P)=-K \sum_{i=1}^{\Omega} P_{i} \log P_{i}
$$

Donde, $\Omega$ es el número de estados posibles en los que puede estar un sistema $S, P_{i}$ es la probabilidad de que el sistema esté en el i-ésimo estado y $K$ es una constante que se establece como $K=\log _{2} 10$. Usando un cambio en la base del logaritmo de 10 a 2, la entropía puede escribirse como:

$$
S(\Omega, P)=-\sum_{i=1}^{\Omega} P_{i} \log _{2} P_{i}
$$

El concepto de entropía para el estudio de grafos fue introducido por Rashevsky (1955), específicamente para el estudio de la topología de los grafos. Köhner (citado en Dehmer y Mowshowitz, 2011) introduce la medida de entropía de grafo para determinar el desempeño de una mejor codificación de mensajes emitidos por una fuente de información donde los símbolos pertenecen a un conjunto finito de vértices en un grafo.

En este trabajo, se usará la entropía de grafo definida por Volchenkov y Blanchard (2008) En el trabajo de Volchenkov y Blanchard se exponen dos formulaciones para la especificación de las probabilidades asociadas con cada vértice de un grafo. La primera usa el concepto de conectividad local para cada vértice. Dicho concepto usa la noción del grado del vértice para determinar que tan «conectado» está éste, localmente hablando. Lo anterior permite construir una distribución de probabilidad definida como sigue. Dado un grafo no dirigido $G(V, E), \forall v_{i} \in V$ se establece la probabilidad:

$$
\pi_{i}=\operatorname{deg}\left(v_{i}\right) / 2 M
$$


Donde, $M=|E|$ es el número total de arcos en el grafo.

Volchenkov y Blanchard (2008) definen también otra distribución de probabilidad basada en la intermediación. Recordando la ecuación (2) en dónde se definió la centralidad de un vértice en función de su intermediación con respecto a los caminos más cortos que pasan por él, la distribución de probabilidad puede escribirse como:

$$
p_{i}=C_{B}\left(v_{i}\right) / C_{B},
$$

Donde $C_{B}=\sum_{i=1}^{N} C_{B}\left(v_{i}\right)$.

La entropía de grafo $H$, de un grafo $G$, se calcula usando la formulación:

$$
H(G, P)=\sum_{i=1}^{N} p_{i} \log _{2}\left(1 / p_{i}\right)
$$

Donde $P$ puede ser la cualquiera de las dos distribuciones de probabilidad antes definidas.

Siguiendo a Volchenkov y Blanchard (2008), sí P es la distribución de probabilidad que es función del grado de los vértices del grafo, la entropía se define como entropía de conectividad. Por otro lado, sí $P$ es la distribución de probabilidad relacionada con el nivel de intermediación de los vértices del grafo, la entropía se define como entropía de centralidad.

La entropía $H(G, P)$ tiene la propiedad de que es aditiva, independientemente de la forma en el grafo se divida en partes. Además, la entropía puede ser calculada como la suma de las entropías parciales de los vértices. Volchenkov y Blanchard (2008) definen la relevancia de cada vértice en términos de su contribución a la entropía o razón de participación en la entropía (entropy participation ratio: EPR), que se define como:

$$
h_{i}=\left(p_{i} / H(G, P)\right) \log _{2}\left(1 / p_{i}\right),
$$

Donde $H(G, P)$ puede ser tanto la entropía de conectividad como la entropía de centralidad.

Midiendo la correlación entre la entropía de conectividad y la entropía de intermediación, es como Volchenkov y Blanchard proponen el cálculo de la inteligibilidad.

A continuación, con el objetivo de ejemplificar el uso del método de Volchenkov y Blanchard se expondrá el estudio que se le realizó a la red de calles que conforman el centro de la ciudad de San Luis Potosí. 
Estudio de LA Red del CEnTRo de la CiUdad de SAN Luis Potosí

La ciudad de San Luis Potosí es una zona metropolitana localizada en el centro norte de México. Fue fundada el 3 de noviembre de 1592 y actualmente está conformada por la ciudad de San Luis Potosí, conurbada con la ciudad de Soledad de Graciano Sánchez y la población de Villa de Reyes. Es la capital del Estado del mismo nombre y su población es de 1,165,379 habitantes, lo que la convierte en la décima primera zona metropolitana más poblada de México. La relevancia de la ciudad se da en distintos rubros de actividades económicas y científicas. En lo industrial, la zona metropolitana es el asentamiento de un importante conjunto de industrias del ramo automotriz que van

FIGURA 2

AGEBS QUE CONFORMAN EL ÁREA DE ESTUDIO

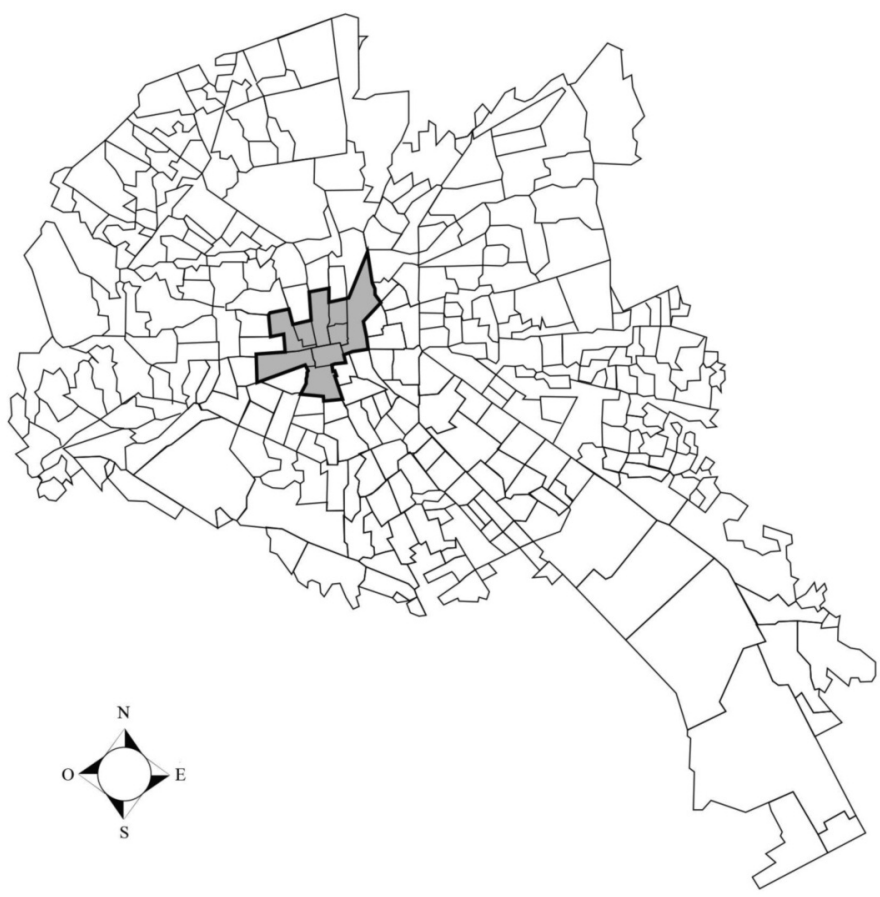

Fuente: elaboración propia. 
desde la construcción de auto partes, hasta el ensamble de automóviles. Así mismo, la ciudad es un importante nodo logístico en el trasiego de mercancías desde y hacia los puertos mexicanos en las costas del Golfo de México y del Pacífico y los Estados Unidos de América. En este rubro se cuenta con un Parque Logístico que es el cuarto recinto aduanero más importante del país. Además, el aeropuerto es sede del centro logístico de una de las mayores empresas de paquetería de México. En lo académico, la ciudad concentra varios laboratorios y centros de investigación de alto perfil. Muchos de los laboratorios e Institutos de Investigación son parte de la Universidad Autónoma de San Luis Potosí y existen tres centros públicos de investigación dependientes del Consejo Nacional de Ciencia y Tecnología.

El centro histórico de la ciudad sigue siendo el centro económico con respecto a comercios al por menor, a pesar de la creación de diversas plazas y zonas comerciales desde los años ochenta hasta la actualidad. Dicho centro está conformado por 175 calles contenidas en 10 AGEBs (Áreas Geográficas Estadísticas Básicas definidas por el Instituto Nacional de Estadística, Geografía e Informática) (figura 2), las cuales contienen 3.278 unidades económicas dedicadas al comercio al por menor, lo que equivale al $17.05 \%$ del comercio al por menor en toda la zona metropolitana.

Para el estudio se construyó un grafo tomando como vértices las calles y como arcos las intersecciones entre ellas (figura 3). La matriz de adyacencia es de $175 \times 175=30,625$ entradas y se calcularon tanto la entropía de conectividad como la entropía de centralidad. Para la construcción de la matriz de adyacencia se usaron los programas Excel y Matlab. Éste último software se usó para el cálculo de la intermediación con el paquete especializado en teoría de grafos. Las calles se codificaron con un número por calle y se construyó un archivo de texto con las calles que intersectan en forma de lista. Dicho archivo se carga sobre Matlab y un algoritmo convierte dicho archivo en una matriz de adyacencia. Dicho algoritmo fue construido por nosotros.

Una vez que se tiene la matriz de adyacencia es trivial el cálculo de la conectividad, ya que la suma de los renglones de la matriz de adyacencia es el grado de cada vértice y con ello se construye de forma inmediata la distribución de probabilidad para la conectividad. El cálculo de la intermediación fue hecho usando un paquete de Matlab de teoría de grafos y fue por ello también fácil dicho trabajo. La manipulación de los resultados se realizó en Excel. Tanto la tabla como los gráficos fueron construidos usando dicho software.

A continuación mostraremos los hallazgos y resultados encontrados. Al aplicar la ecuación (8) considerando la conectividad de los 175 nodos que conforman la red encontramos que la entropía de conectividad es 6.977. Al 
FIGURA 3

GRAFO CORRESPONDIENTE A LAS 175 CALLES DEL CENTRO DE LA CIUDAD DE SAN LUIS POTOSÍ

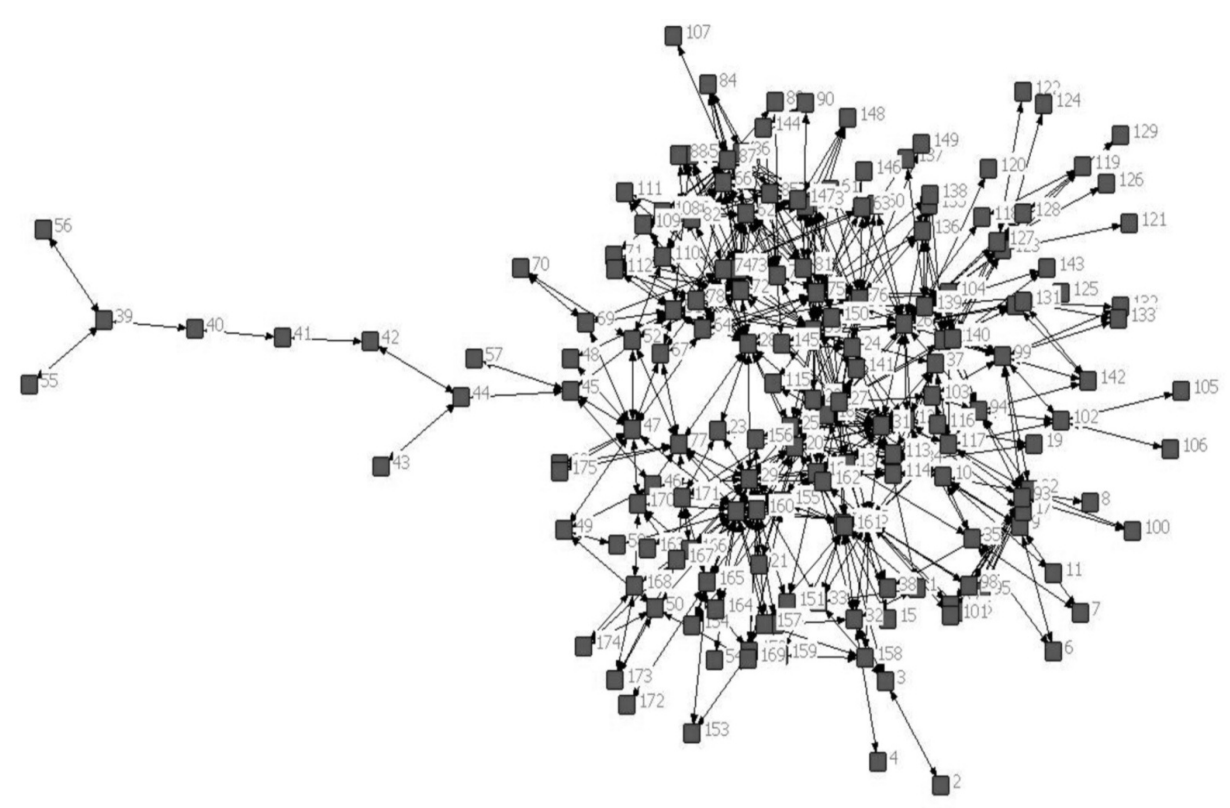

Fuente: elaboración propia.

aplicar la ecuación (8) considerando la distribución de la centralidad para los mismos 175 nodos encontramos que la entropía de centralidad es de 5.6441. Nótese que el valor de la entropía para el grafo totalmente conexo es $\log _{2} \mathrm{~N}=$ $\log _{2}(175)=7.451211$. Dicho valor representa la situación en el que todos los nodos son adyacentes unos de otros y los caminos más cortos son de tamaño 1. Lo anterior lo podemos interpretar de la siguiente manera, con respecto a la conectividad total la red de calles del centro histórico de la ciudad de San Luis Potosí presenta un $93.63 \%$ de conectividad entre si. Por el contrario solo el 75.75\% de las calles funcionan como concentradoras o ejes de actividad peatonal al ser pasos obligados para llegar a otras calles.

Se usó la ecuación (9) para calcular la razón de participación en la entropía (EPR) tanto para la conectividad como para la centralidad. Los resultados se pueden observar en el diagrama de dispersión de la figura 4. 
FIGURA 4

\section{RESULTADOS DE CALCULAR LAS MEDIDAS ESTRUCTURALES PARA LA RED DE CALLES DEL CENTRO DE LA CIUDAD DE SAN LUIS POTOSÍ}

Diagrama de dispersión para las 175 calles

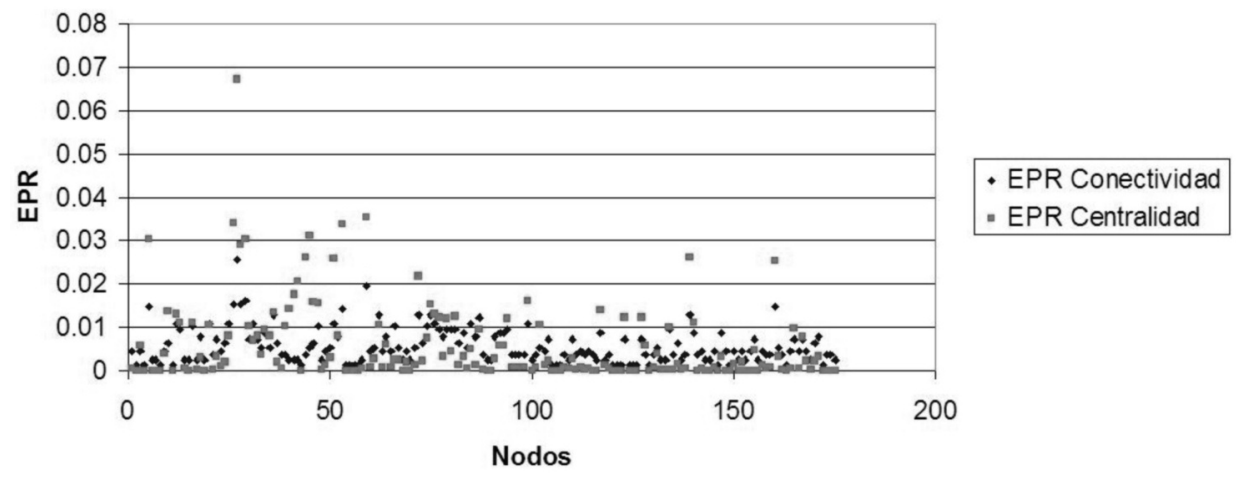

Fuente: elaboración propia.

Del estudio de la EPR podemos observar la existencia de 33 calles (nodos) con EPR de conectividad alta con respecto al resto de los nodos. Lo que deja ver que el $81.14 \%$ de las calles están poco conectadas a nivel local. Este resultado contrasta con nuestra primera interpretación basada en la entropía global y muestra la utilidad del desglose de la entropía para analizar de forma fina la estructura del espacio urbano. El promedio de conectividad, es decir el grado promedio es de 5.77 nodos interconectados a nivel local para cada nodo en la red. Sin embargo, la desviación estándar es de 5.25. El grado mínimo es 1 y el grado máximo es 38. Existen 23 calles (nodos) con grado de segregación máximo ya que su grado es solo 1 .

Con respecto al EPR de centralidad encontramos que el $25.76 \%$ de los nodos no contribuyen a la centralidad, su valor es 0 . Esto significa que por dichos nodos no pasa ningún camino que interconecte con los demás nodos y los podemos interpretar como calles marginales. Solo 14 nodos ( $8 \%$ ) tienen alto grado de centralidad (mayor a 0.02 ). El nodo 27, correspondiente a la calle de «Reforma» es el que tiene el mayor valor de centralidad. Hay que hacer notar que este nodo es el que presenta mayor grado de conectividad. Lo anterior lo interpretamos como una red de calles con un alto potencial de servir como centros de actividad con un pequeño subconjunto que sir- 
ven de ejes concentradores o pasos obligados dentro de la movilidad peatonal en la red.

Para calcular la inteligibilidad de la red se procedió a calcular el grado de correlación entre la EPR de conectividad y la EPR de centralidad. En nuestro caso, el EPR de conectividad y el EPR de centralidad tienen un índice de correlación de Pearson de 0.79785949 , lo que indica que ambos índices están correlacionados de forma positiva y por ende hay inteligibilidad en el entorno urbano estudiado (figura 5).

Dado los resultados obtenidos podemos hablar de que la estructura del «tejido urbano» bajo estudio presenta complejidad organizada. Lo anterior se interpreta en el marco de la cognición del espacio como que dicho espacio urbano es fácilmente aprensible bajo las condiciones de percepción de la estructura de las trayectorias que se realizan en el mismo. Lo anterior implica que la complejidad se simplifica ante la organización jerárquica del espacio en la mente del peatón. Para éste, la necesidad de tener muchas alternativas de trayectorias o recorridos, y la capacidad de compararlas mentalmente, es la clave del pensamiento razonado.

Consideramos que el método propuesto por Volchenkov y Blanchard resulta más adecuado que el propuesto por Hillier, en lo que se refiere a las me-

FIGURA 5

DIAGRAMA DE DISPERSIÓN EN DONDE SE MUESTRA LA CORRELACIÓN ENTRE LA EPR DE CONECTIVIDAD Y LA EPR DE CENTRALIDAD

\section{Correlación}

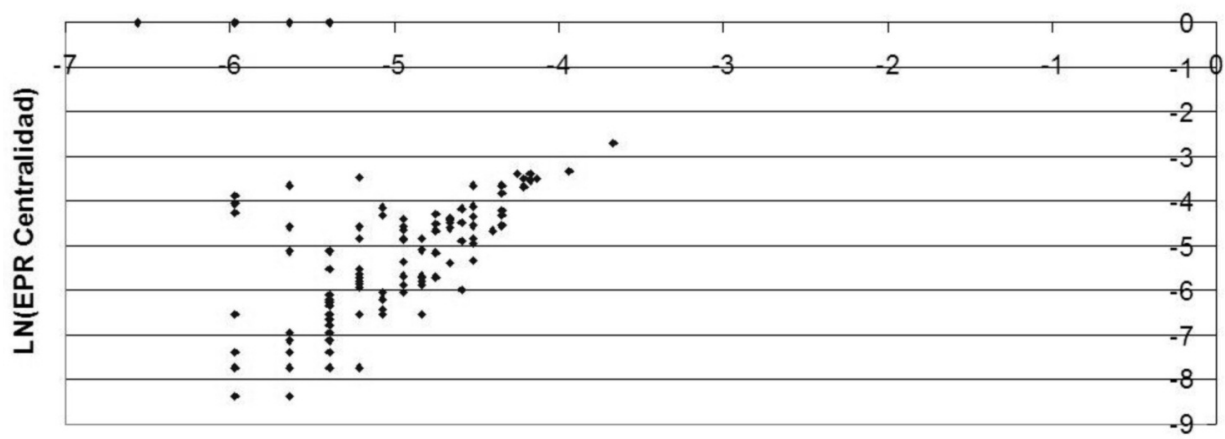

LN(EPR Conectividad)

Fuente: elaboración propia. 
didas globales y locales de la estructura del entorno urbano, las cuales son interpretadas a la luz de la teoría de grafos. Por ejemplo, en el cálculo de Hillier la medición de la profundidad media implica la medición de las distancias entre los centros de cada segmento de recta del mapa axial. Esta medida global se sustituye por el grado de intermediación de los vértices con respecto a recorridos sobre el grafo, lo que se puede interpretar fácilmente como la frecuencia en que un peatón pasaría por ciertas calles haciendo caminatas aleatorias.

Queremos hacer notar que el método propuesto por Volchenkov y Blanchard permite analizar a detalle la función de cada calle dentro del sistema. Sí solo usáramos las entropías globales los resultados son muy diferentes. Este nivel de detalle es el que consideramos de gran utilidad para nuestro análisis.

Otra ventaja que encontramos es que el método propuesto por Volchenkov y Blanchard no necesita el uso de Sistemas de Información Geográfica y paquetes especializados en Space Syntax. De esta forma sorteamos la limitante de la construcción del mapa axial y del cálculo tanto de la profundidad como de la integración.

Por último queremos resaltar que este estudio ilustra la pertinencia de los conceptos de contenido de información y entropía para medir la parte cognoscible de las estructuras espaciales.

Fecha de recepción: 17 de febrero de 2012.

Fecha de aceptación: 27 de junio de 2013.

\section{BibLiografíA}

Blanchard, P. y Volchenkov, D. (2010): Mathematical analysis of urban spatial networks. Berlin, Springer-Verlag.

Dehmer M. and Mowshowitz, A. (2011): "A history of graph entropy measures". Information Sciences, 181/1, pp. 57-78.

Freeman, L. C. (1977): "A set of measures of centrality based on betweenness". Sociometry, 40/1, pp. 35-41.

Haq, S. and Girotto, S. (2003): "Ability and intelligibility: Wayfinding and environmental cognition in the designed environment", en: Proceedings. 4th International Space Syntax Symposium London, 17-19 June, 2003. Disponible en línea: http://www.spacesyntax.net/symposia-archive/SSS4/fullpapers/68Haq-Girottopaper.pdf (Fecha de consulta: 27/10/2014).

Hillier, B. (1996): Space is the machine. Cambridge, Cambridge University Press.

Hillier, B. and Hanson, J. (1984): The social logic of space. Cambridge, Cambridge University Press. 
Lynch, K. (1997): The image of the city. Cambridge, Massachusetts, Massachusetts Institute of Technology Press.

Rashevsky, N. (1955): "Life information theory and topology". Bulletin of Mathematical Biophysics, 17, pp. 229-235.

Volchenkov D. and Blanchard, P. (2008): "Discovering important nodes through graph entropy encoded in urban space syntax". Disponible en: http://arxiv.org/ PS_cache/arxiv/pdf/0709/0709.4415v1.pdf (Fecha de consulta: 22/10/2014).

\title{
RESUMEN
}

Dentro de los estudios de cognición del espacio, Hillier (1996) propuso el concepto de inteligibilidad como una propiedad del espacio que permite que éste sea comprendido por las personas que lo transitan y/o habitan. Hillier, dentro de la teoría de la sintaxis del espacio, propone que la inteligibilidad es la correlación entre una variable local y una variable global. Basados en lo anterior, Volchenkov y Blanchard (2008) han propuesto una forma de medir la inteligibilidad fuera del contexto técnico de la teoría de la sintaxis del espacio. Este trabajo recupera la discusión teórica-metodológica del trabajo de Hillier y expone el método de Volchenkov y Blanchard. Como ejemplo del mismo se midió la inteligibilidad del centro histórico de la ciudad de San Luis Potosí, México. Se encontró que dicho espacio tiene una inteligibilidad alta según el método propuesto por Volchenkov y Blanchard.

Palabras Clave: inteligibilidad del espacio; entropía de grafos; sintaxis del espacio.

\begin{abstract}
Within space cognition studies, Hillier (1996) proposed the concept of intelligibility as a property of space that allows it to be understood by people that either live in or transit through that space. Hillier, in the theory of space syntax, suggests that intelligibility is the correlation between a local variable and a global variable. Basing themselves on the above, Volchenkov and Blanchard (2008) proposed a way to measure intelligibility outside the technical context of the theory of space syntax. This paper returns to theoretical and methodological discussion of Hillier's work and explains the method proposed by Volchenkov and Blanchard. As an example, the intelligibility of the historic center of the city of San Luis Potosi, Mexico, was measured. It was found that this space has a high intelligibility in the terms of the method proposed by Volchenkov and Blanchard.
\end{abstract}

KEY WORDS: intelligibility of space; graph entropy; space syntax.

\section{RÉSUMÉ}

Dans le cadre des études de la cognition spatiale, Hillier (1996) a proposé le concept d'intelligibilité comme une propriété de l'espace permettant que celui-ci soit compris par les personnes qui y transitent et/ou l'habitent. Hillier propose, dans le contexte de 
la théorie de la syntaxe spatiale, que l'intelligibilité est la corrélation existante entre une variable locale et une variable globale. Conformément à cette théorie, Volchenkov et Blanchard (2008) ont proposé une méthode pour quantifier l'intelligibilité en dehors du contexte technique de la théorie de la syntaxe spatiale. Le travail ci-présent reprend la discussion théorique et méthodologique du travail de Hillier et expose la méthode de Volchenkov et Blanchard. À titre d'exemple, l'intelligibilité du centre historique de la ville de San Luis Potosí, au Mexique, est quantifiée. Le résultat est que cet espace possède un haut niveau d'intelligibilité selon la méthode proposée par Volchenkov et Blanchard.

Mots CLÉs: intelligibilité de l'espace; entropie des graphes; syntaxe spatiale. 